\title{
El urbanismo sin interés social
}

\section{Juliana López Vergarał}

DOI: 10.24142/indis.v6n11a2

Recibido: 2 de abril de 2020 - Aceptado: 5 de junio de 2020 - Publicado: 30 de agosto de 2020

\section{Introducción}

Palabras clave: Neoliberalismo, urbanismo, marketing urbano, participación

La identidad de un ciudadano se construye; las circunstancias que lo rodean y el entorno en el que crece lo forman, lo sincronizan con el espacio en que habita, incluyendo a las personas con las que convive y, de esa manera, se siente un miembro de la ciudad, con las costumbres y visiones que tiene de ese entorno. Una relación que ha de ser entendida como parte de las distintas dinámicas de vivencia que se practican dentro de la ciudad y en tanto eso, ha de ser respetada.

Las comunidades desde su consolidación se han encaminado a gestionar proyectos en virtud de resolver las necesidades y problemáticas que dentro de esta se vivan. Y es así como al buscar la mayor eficiencia y lo más apropiado para esta, se construye la identidad de los sujetos, de acuerdo con la forma en que la sociedad donde se desarrollan soluciona los conflictos.

Siguiendo esta lógica, los sujetos con el tiempo cimientan su identidad en un entorno que los identifica, uno que tiene significado para sí mismos, dándoles autonomía, participación mediante el desarrollo de estilos de vida y la solución de las pro-

17 Estudiante de noveno semestre de la Facultad de Derecho de la Universidad Autónoma Latinoamericana, UNAULA. Integrante del semillero Cartografiar el Derecho. Correo: juliana.lopez6214@unaula.edu.co 
blemáticas; es entonces, cuando el territorio adquiere significado para éstos, porque las relaciones sociales no responden a una forma estereotipada del espacio sino al tiempo de convivencia con este, en el cual han cimentado su historia.

Actualmente, la tensión social se desarrolla ante un reposicionamiento de una institución de gran poder como lo es el mercado, el cual ha asumido el rol principal para dirigir las relaciones sociales, mediante la regulación estricta de las relaciones intersubjetivas, cuya finalidad es lograr la desvinculación de los sujetos con el territorio, desaparecer la identidad construida por el habitante con su lugar de convivencia, siendo permeado por un discurso en pro del desarrollo y la innovación. Se está en presencia de un modelo perpetuado por el mismo Derecho y un Estado que solo funciona para el bienestar de la economía.

El neoliberalismo fundamenta este nuevo panorama en "un mercado abierto, competitivo y sin regulación como el mecanismo más eficiente para el desarrollo económico" (Peck, Theodore y Brenner, 2009) que ha terminado por desencadenar mayores problemáticas como la marginalización y la exclusión dentro de las ciudades.

El conflicto por desarrollar puntualmente nace a partir de las intervenciones físicas en el territorio, desarrolladas por la Alcaldía de Medellín como institucionalidad estatal local. Dicha intervención de la administración en las relaciones que se crean entre los comunes, ha redimensionado la realidad de los pobladores hacia un territorio desconocido, desprovisto de cualquier sentido de pertenencia para ellos; ya que mediante los procesos de reasentamiento, más allá de una rehabilitación profunda de los espacios, se ha convertido en un intento por reconquistar aquellos sitios que vivieron la ausencia estatal durante años y siguiendo la lógica de la conquista, se termina destruyendo aquello que no encaja en su razonamiento; los habitantes reasentados por la Administración no son más que sujetos que no encajan en la fórmula de la ciudad neoliberal.

La ciudad de Medellín, una de las ciudades más innovadoras, ha recurrido a políticas competitivas, mediante el marketing urbano y la ostentación arquitectónica. En gran medida, esta metrópolis ha permitido que se deje en segundo plano al sujeto, mediante "inversiones selectivas y acupunturas urbanas" (Brand, 2013), las cuales apuntan al desarrollo de estrategias diseñadas para elegir zonas de la ciudad (muy específicas) que con la correcta inversión, pueden ser generadoras de mayor atracción en el contexto internacional (una manera de pensar a la ciudad, como un 
producto de exportación), generando menoscabo para muchos reasentados al perder su sincronía con el territorio, es decir, la misma identidad que tienen los pobladores como ciudadanos con el espacio que habitan.

Esta problemática, implica indagar por la implementación del modelo de urbanismo social en Medellín, especialmente, desde la administración del alcalde Sergio Fajardo Valderrama (2004-2007), a partir de los procesos de intervención y reasentamiento desde ciertas obras de modificación urbanística como el Plan Parcial Naranjal; propuesta que, en principio, pretendió abordar las problemáticas sociales de los habitantes de la zona, siendo pensados como lugares marginados, que no encajan en el proyecto de una Medellín con una visión de transformación territorial, moderna, pacífica y organizada; por ende, se debía realizar la intervención necesaria mediante el sometimiento de la población a las políticas públicas. La mencionada intervención terminó generando una crisis de identidad, ya que la realidad que construyó la Administración de Medellín se cimentó sobre la destrucción del entorno de los pobladores, teniendo éstos que adaptarse a una nueva realidad, donde finalmente, resultaron siendo los perjudicados con la renovación del territorio. Es durante estos años cuando el discurso neoliberal logra empoderarse y determinarse como la manera más idónea de administrar a Medellín.

Es necesario plantearse ihan sido los métodos usados por la Administración de Medellín, para solucionar la situación, los que han terminado por desencadenar nuevas problemáticas para los sujetos del plan parcial?

Son posibles consecuencias cuando se olvida que, para la implementación de nuevas políticas, es necesario tener en cuenta hacia quiénes son dirigidas y que no han de quedarse en simples estadísticas, que, a grandes rasgos, no pueden especificar las particularidades tan complejas que tiene una sociedad. Y es así como una decisión puede hacer de toda una población, parte de una ecuación que los ve como productos a restar.

\section{Urbanismo neoliberal}

\section{Acerca del neoliberalismo}

Durante las últimas décadas, la corriente neoliberal ha desarrollado políticas para la reconfiguración de las ciudades, las cuales responden a una rentabilización de la ciudad, la explotación de lo urbano como un producto de exportación, promovidas por 
el nuevo reposicionamiento del mercado: la institución encargada y legitimada para dirigir las relaciones sociales y la asignación de recursos.

Como sostiene Michael Janoschka, "el neoliberalismo está lejos de ser una corriente en agonía; es un proceso que apenas está en curso y tiene como propósito la transformación socioespacial de la ciudad"18. Con mayor fuerza en América Latina, se ha constituido como la estructura perfecta según la lógica que vende el sector privado, donde todo aquello que esté por fuera del neoliberalismo es "irracional". Este panorama expone la caída de la figura del Estado-Nación como el actor económico principal, y reconoce que los actores sociales, políticos y económicos han sido alterados y, que hoy en día, es la ciudad la que se construye ( 0 a la que construyen) como un escenario privilegiado de capitalización neoliberal. El Estado no es más que el encargado de garantizar la dinámica competitiva de la economía, bajo los márgenes de la eficacia y la eficiencia"19.

Se plantea una forma de administración de la urbe "a partir de los criterios empresariales"20, que giran alrededor del marketing urbano, la rentabilización de la ciudad, el embellecimiento de esta y la venta de una hiperrealidad al mercado internacional. Estableciéndose al mejor estilo de un Estado gerencial, pero en cabeza de las ciudades.

Sin embargo, la cuestión radica en cuál ha sido la razón por la que la corriente imperante ha centrado su desarrollo y adaptación en la figura aparentemente pequeña de la ciudad.

La urbe, entendida desde los términos de optimización y potencialización del mercado, se enmarca como estructura organizacional compleja, que entraña relaciones y problemáticas entre los sujetos; y siendo el consumo la referencia básica de la corriente capitalista, el campo de las relaciones sociales se reduce y sobre estas se cimientan las relaciones mercantiles, cuya naturaleza sí responde a la racionalidad del neoliberalismo.

18 Hidalgo, R., \& Janoschka, M. (Eds.). (2014). La ciudad neoliberal: gentrificación y exclusión en Santiago de Chile, Buenos Aires, Ciudad de México y Madrid. Rodrigo Hidalgo y Michael Janoschka.

19 Noreña, J. P. S. (2017). Marketing urbano, forma de gobierno neoliberal en la ciudad de Medellín. Iconofacto, 12(19), 124-153.

20 Hidalgo, R., \& Janoschka, M. (Eds.). (2014). La ciudad neoliberal: gentrificación y exclusión en Santiago de Chile, Buenos Aires, Ciudad de México y Madrid. Rodrigo Hidalgo y Michael Janoschka. 
La expansión de la economía es la manera más eficiente de actuar del Estado gerencial, en pro de permitirle al mercado intervenir en todos los ámbitos de la vida, "ya no se limitan al escenario del intercambio de bienes y servicios, sino que también se entremezcla en las creencias, comportamientos sociales, políticos, religiosos (...) dan forma a la vida de los ciudadanos" ${ }^{21}$. Se fundamenta todo un sistema alrededor de una sola corriente, de una sola ideología, porque se cree que es la única capaz de traer el desarrollo.

Esta ideología económica tiene la particularidad de interesarse, en primera medida, por las trayectorias del lugar en el cual pretende insertarse, la elaboración de sus políticas no es al azar. El concepto de "neoliberalizar" un territorio, implica también determinar cómo ha de ser la conexión, respondiendo a la creación de políticas específicas para cada lugar. La corriente reconoce los diferentes tejidos de las sociedades y se adapta a ellas, para lograr una reestructuración silenciosa y profunda.

El neoliberalismo siendo uno solo, respeta las particularidades para que sea más fácil su asimilación dentro del sistema, logra ser aceptado (promovido y muchas veces ovacionado) para vincularse como la mejor opción para resolver los disensos sociales (elaborada y diseñada a partir de su propia existencia como principal regulador de las relaciones intersubjetivas).

Como lo expone Pradilla Cobos (2014), esta situación no es tan homogénea como plantean los analistas e investigadores de los países hegemónicos. A diferencia del aparato Estatal (el cual concibe su territorio como un espacio homogéneo y sin irregularidades), la corriente económica ha logrado ajustarse minuciosamente a cada escenario donde pretende invertir; se adapta y logra manipular aquellas particularidades a su favor.

El modelo de ciudad neoliberal no parte de un estudio general y abstracto de la urbe. La misma corriente se encarga de seleccionar y delimitar qué espacios dentro de la ciudad son óptimos para legitimar su intervención; en otras palabras, tiene en cuenta las problemáticas del espacio, la historia del territorio, las interacciones entre los sujetos, determinando así qué tan rentable es invertir en este; una inversión selec-

21 Noreña, J. P. S. (2017). Marketing urbano, forma de gobierno neoliberal en la ciudad de Medellín. Iconofacto, 12(19), 124-153. 
tiva para reimaginar un espacio de la ciudad en favor del desarrollo, aunque para ello deba reducirlo y dejar a los mismos pobladores por fuera de su lógica.

\section{Los sujetos dentro de la ciudad}

Si partimos del entendido según el cual toda contraposición de pensamientos genera un conflicto, cuyas manifestaciones pueden ser tanto intelectuales como materiales. Hoy en día, estamos frente a una tensión respecto de quiénes defienden las políticas neoliberales como oportunidades de competencia y desarrollo al nivel del último siglo y quiénes ven en ellas una realidad distorsionada con lógicas desinteresadas por lo público. Sin embargo, han sido notorias las ventajas en las que se fundamenta el neoliberalismo mediante sus prácticas, ya que estas cuentan con mayor aceptación por parte de los mismos gobiernos.

El sistema actúa mediante desposesiones, pero no solo en el nivel material, hablamos de la perpetuación teórica y práctica de alienación del individuo, en busca de desnaturalizar su identidad con el espacio e imponerle una estigmatización, para que sea más fácilmente identificable dentro de la producción de la segregación social; volverlo un producto financiable.

La apuesta está en vincular al sujeto con los conceptos que aportan lógica a los sistemas del mercado. Se habla de una situación en la que es más fácil crear un falso sentido de pertenencia, como un concepto abstracto propio del neoliberalismo, que intentar crear 0 en muchos casos recuperar la conexión con lo ciudadano.

El sujeto dentro de la urbe nace con el propósito de enriquecerse del progreso de esta, mientras que, el sujeto de la periferia, los hacinados, los que no encajan con el modelo de desarrollo, deben ser transformados, pero no en sujetos con oportunidades como el primero, sino en obreros.

La ciudad compacta ${ }^{22}$, incentiva la producción de clases sociales, poniendo en la cúspide a quienes despojan (atendiendo a las novedosas y desarrolladas formas neoliberales) y en la base, cargando con todo el peso del cambio, a quienes son desposeídos. Además, aquellos que no puedan adaptarse a esta lógica, pueden llegar a perder su existencia dentro de la ciudad compacta, por absurdo que suene; porque

22 Abramo, P. (2012). La ciudad com-fusa: mercado y producción de la estructura urbana en las grandes metrópolis latinoamericanas. EURE (Santiago), 38(114), 35-69. 
para el mercado se convierte en un dato, en una enorme estadística que no debe ser tenido en cuenta.

Para Laval y Dadot, esta nueva lógica considera a las poblaciones y a sus individuos, desde el punto más estrecho, es decir, desde su contribución y su coste. Debido a esta mentalidad, no importa los métodos que hayan de efectuarse para captar la atención del público, cómo ha de convencerse a este, de qué le es más conveniente. Darle al público lo que quiere, aunque no sepa eso que quiere, le ha permitido a la corriente tener mayor conocimiento del individuo, de su potencial consumidor; y es por ello que le es fácil rediseñar y moldear su discurso según los deseos, cualesquiera que sean para construir un estereotipo de ciudad, que cubre a la ciudad vivida con estructuras vacías porque las personas no se identifican con esos nuevos espacios, hay una total desincronía con el territorio, pero esa problemática ya no le concierne al neoliberalismo.

\section{Generador de expulsiones intraurbanos en Medellín}

En palabras de Borja, "(...) mediante políticas sectoriales y cortoplacistas acaba sometiéndose a la lógica segregadora y excluyente del mercado y contribuye en muchos casos a la disolución de lo ciudadano. A lo que gobernantes (derechas e izquierdas confundidas) y grandes empresas añaden en nombre de la competitividad y del marketing urbano la ostentación arquitectónica, el neomonumentalismo de exportación, que banaliza la ciudad y aliena a los ciudadanos, puesto que en muchos casos esta arquitectura de autor parece destinada a provocar sentimientos de expropiación, en vez de la identificación o la emoción integradoras. El sentimiento de desposesión es hoy perceptible en las ciudades metropolitanas".

Un sistema de desmantelamiento de lo público, por la apertura a la iniciativa privada de los servicios que antes proporcionaba de manera principal el Estado, fortaleciendo así las prácticas sociales dominadas por el individualismo y el consumismo, para profundizar la segmentación social.

Por ello es común observar en las grandes ciudades territorios de supervivencia, donde los sujetos luchan por integrarse a la ciudad, tratando de superar la constante competencia, producto de la marginalización y sectorización. Lo cual ha generado una ciudad polarizada, de paisajes fragmentados, con espacios públicos perdidos y una comunidad que pierde, cada vez más rápido, su posición dentro de esta. 
En la ciudad de Medellín, la corriente del urbanismo con enfoque social se fortalece y se convierte en el discurso institucional, llevando, por una de las caras de la moneda, una lógica de interés en el colectivo, interés en lo público y respeto por los moradores de los distintos lugares a intervenir23; y por la otra, lo que viene por debajo de la expropiación, está la lógica internacional de la ciudad innovadora, de la ciudad producto de exportación, que en el marco de lo privado se ve como una gran empresa que hay que remodelar constantemente. La alcaldía ha diseñado planes, que pretenden intervenir aquellos lugares que durante años fueron el eterno rechazo de la administración, por la informalidad en la que vivían, pero que, al ser seleccionadas, mediante las acupunturas urbanas promovidas por la corriente neoliberal, pasan del anonimato a ser una de las grandes expectativas a mostrar cuando se termine, al mercado mundial.

Para entender la dinámica neoliberal, y el porqué de la intervención de sectores específicos, como lo fue Naranjal, es necesario conocer la realidad social de territorio y la razón por la cual, se tornó en un espacio ideal para ser partícipe de la renovación de la ciudad de Medellín. Es necesario plantearnos en qué se beneficia el desarrollo de la ciudad con la renovación de Naranjal.

En primer lugar, el sector de Naranjal se vio fuertemente afectado con la crisis industrial de 1970, y habiendo sido pensado como un modelo de industrialización para la ciudad, el fracaso de este tuvo como consecuencia que, al llegar influencias del narcotráfico y la criminalidad, se volviese un espacio sin legalidad, sin presencia estatal.

Desde el Estatuto Municipal de Planeación y Urbanismo de Medellín de 1990, el sector de Naranjal se destina, preferentemente, para la ejecución de proyectos de vivienda, asociado con el propósito de transformar a la ciudad en territorio de servicios, convirtiéndose el sector en un área de renovación inminente con el fin de compatibilizarla con el sector residencial Suramericana y con las obras del Metro y lo proyectado para recuperar el río (Cuesta, 2016).

Y es con este último factor que la institucionalidad justifica principalmente su intervención, al fin y al cabo, el Plan Parcial se convirtió en la manera de legitimar el Plan Parcial de Renovación Urbana para Naranjal, adoptado mediante el decreto

23 Artículos 39 de la ley $9^{\circ}$ de 1989 y 119 de la ley 388 de 1997. 
1284 de 2000, el cual contaba con lagunas normativas que permitieron dejar de lado y desprotegidos a los moradores del territorio (Cuesta, 2016).

Promovido por la Alcaldía de Medellín, como forma de legalizar el accionar de la Administración, este decreto asegura y protege la intervención del verdadero actor privilegiado, por encima de los pobladores: el mercado inmobiliario.

Aunque las acciones de la alcaldía se movían dentro de un discurso de "servicios con la vivienda multifamiliar, destinando para esta última, las porciones internas más tranquilas y con mejores condiciones ambientales", el proyecto terminó por incentivar la parte comercial inmobiliaria, dotándola de los beneficios que el punto pudo ofrecerle; solo cuestiones de estrategia.

La ubicación estratégica del sector se constituye en la principal justificación de la Alcaldía para desarrollar políica, económica y jurídicamente el Plan Parcial. "Naranjal se encuentra ubicado en cercanías al sistema de transporte de gran capacidad Metro de Medellín y al barrio Suramericana, considerado referente arquitectónico y social de la ciudad que posee una consolidación importante por la construcción en altura y la existencia de un amplio sector de comercio y servicios, especialmente financieros. En el futuro cercano el sector también tendrá una cercanía importante con Parques del río II dentro de la Autopista Sur, lo que permite una mejor ubicación de los posibles desarrollos en términos de afianzamiento del mercado inmobiliario".

Realmente, el territorio sufrió un impacto de la administración, dado el incorrecto y poco profundo desarrollo que se hizo de uno de los principios sobre los cuales se cimentó el plan, que era crear los mecanismos necesarios para que hubiese equidad entre la protección de los moradores y el restablecimiento del lugar.

Como se decía al principio, la corriente económica que se adoptó en la ciudad de Medellín fue legitimada por el mismo derecho para el menoscabo de la convivencia de toda una comunidad; ya que, si bien el Plan Parcial se encaminó a servir a los intereses del colectivo, la gestión social realizada por la administración se desinteresó de las subjetividades de los habitantes del sector y se guio únicamente por el desarrollo altamente competitivo, que venía detrás del sector inmobiliario que quería crecer en ese espacio. Lo hace mediante el mecanismo por excelencia de la institucionalidad para poder reconstruir territorios: la expropiación administrativa. 
Lo denso del asunto, lo constituye la evidencia que deja este tipo de planes que pretenden construir y desarrollar ciudad, destruyendo configuraciones de vida que no siempre tienen que estar ofrecidas por la entidad estatal.

No es ajeno que las relaciones culturales y sociales de cómo se constituye el sentido de pertenencia con un territorio y el arraigo con éste, no caben en porcentajes y, por ende, son considerados elementos de menor trascendencia para la alcaldía, haciendo más difícil el reasentamiento.

En cuanto al arraigo en el sector, se identificó el inicio de un fuerte arraigo por parte de la mayoría de la población, llevando el 60\% de la población más de seis años en el sector, el 21\% entre dos y cinco años y apenas el 18\% de la población lleva menos de un año de permanencia (Cuesta, 2016).

Como bien se ha dicho, la inversión selectiva, termina por excluir, embellecer y revalorizar las áreas más rentables para el sector privado; desencadenando nuevas problemáticas para los pobladores que ven cómo su territorio es neoliberalizado.

El carácter desigual de la corriente neoliberal ha permitido que se deje atrás a una ciudad vivida, por una ciudad marketing; nuevas formas de polarización mediante la competencia. La intervención neoliberal ha transformado a la ciudad, en una ciudad violenta, provocada por la producción de la desigualdad social como resultado del mismo orden económico y, por otra parte, la violencia "genera una estigmatización territorial de los espacios marginados, aumentando la desertificación institucional y profundizando el cercamiento como medio de control social y espacial"24.

En Medellín, frente al Plan Parcial Naranjal, el mismo proceso de reasentamiento, reconoce que, de alguna u otra forma, existen circunstancias que la administración de la ciudad no puede dejar de lado como la producción de ausencia, las desvinculaciones, la destrucción del espacio y la pérdida de identidad que ha provocado la expulsión. Para los pobladores reasentados no basta con una legitimación normativa del actuar del Estado, porque sí bien, se generaron "espacios para que los sujetos intervinieran con su participación", se tornaron ineficientes para la comunidad y al final, frente a las políticas públicas, éstos no tienen una cooperación significativa junto con la entidad estatal, dejándolos completamente por fuera del proyecto que pretende despojarlos de su espacio.

Luna, F. G. (2013). Espacialización de la violencia en las ciudades latinoamericanas: una aproximación teórica. Cuadernos de geografía, 22(1), 169-186. 


\section{Bibliografía}

Borja, J. (2014). Ciudad, urbanismo y clases sociales. Sin Permiso, 27.

Cobos, E. P. (2014). La ciudad capitalista en el patrón neoliberal de acumulación en América Latina. Cuadernos Metrópole, 16(31), 37-60.

Cuesta Gómez, 0. Formas de intervención del hábitat en Medellín. de los procesos de mejoramiento de barrios a los planes parciales de renovación, 1960-2015 (Doctoral dissertation, Universidad Nacional de Colombia-Sede Medellín).

Hidalgo, R., \& Janoschka, M. (Eds.). (2014). La ciudad neoliberal: gentrificación y exclusión en Santiago de Chile, Buenos Aires, Ciudad de México y Madrid. Rodrigo Hidalgo y Michael Janoschka.

Janoschka, Michael (2002). "El nuevo modelo de la ciudad latinoamericana: fragmentación y privatización” en Eure vol. XXVII, núm. 85, páginas 11-29.

Montoya-Restrepo, N. (2014). Urbanismo social en Medellín: una aproximación a partir de la utilización estratégica de los derechos. Estudios políticos, (45), 205-222.

Noreña, J. P. S. (2017). Marketing urbano, forma de gobierno neoliberal en la ciudad de Medellín. Iconofacto, 12(19), 124-153.

Rey, M. T. (2010). Después de la globalización neoliberal. ¿Qué Estado en América Latina? Cuadernos de Pensamiento Crítico Latinoamericano, 32.

Smith, N. (2009). ¿Ciudades después del neoliberalismo? Smith, N.; Observatorio Metropolitano; Rolnik, R.; Ross, A. E. DAVIS, M. (eds.). Después del neoliberalismo: ciudades y caos sistémico. Barcelona, Universidad Autónoma de Barcelona, 9-30. 